\title{
Examination of Possible Land Use Patterns in a Port City Model
}

\author{
Hiroyuki KoIDE*
}

\section{Introduction}

During the past two decades there have been considerable efforts made in urban economics to extend and generalize the initial works of Alonso (1964) and Muth (1969). ${ }^{1}$ One such effort was to remove the locational fixities typically assumed in a monocentric model of a city and to determine simultaneously the equilibrium locations of all the agents in the urban economy. Mills (1972) is the forerunner of this general equilibrium approach in a setting of a port city, which slightly extends the traditional Alonso-Muth type model by treating production activities explicitly. Using a simple coefficient-model, Mills suggested an integrated solution in which firms and households are mixed together, as well as segregated one which has a usual monocentric land use pattern. Later works in the literature attempt to remove the ad hoc assumption regarding the monocentric land use pattern and to determine a general equilibrium urban configuration through the simultaneous determination of rent and wage curves. Of course, to avoid the trivial solution of a uniform distribution of activities, these works introduce in their models some kind of internal forces which will produce the tendency of agents to cluster. ${ }^{2}$

From this trend, the simple framework of Alonso-Muth type models seems to be discarded because of their ad hoc assumption regarding monocentricity. Indeed, most of the Alonso-Muth type models narrowed their scope of analysis by assuming a priori the existence of a central business district, or lacked thorough investigation into the implications of the models due to the restrictive functional forms adopted (e.g., fixed-coefficient production technology, fixed consumption of land by households, etc.). However, a complete characterization of a port city model $^{3}$ will reveal its real explanatory power as well as its limitations. If we adopt more general functional forms, the port city model may generate more urban spatial structures than those models with complicated price or

* Faculty of International Studies and Business, Kyushu International University

1. See Wheaton (1979) for the earlier works of the new urban economics.

2. See Beckmann (1976), Ogawa and Fujita (1980), Fujita and Ogawa (1982), Imai (1982), O'Hara (1977), and Tauchen and Witte $(1983,1984)$. Fujita (1988) gives a survey on price and nonprice interaction models.

3. Goldstein and Moses (1975), and Schweizer and Varaiya (1976) studied the optimal location of production activities with a Leontief-type technology in the setting of a port city. But their analyses do not contain households' residential choice, which is important in our model. 
nonprice interactions among agents. Thus, thorough examination of the port city model also will help us appreciate properly the role of price and nonprice interactions among agents in modeling the urban economy.

The purpose of this paper is to investigate the possible land use patterns which will emerge due to the proximity to a transport node. For this, we formulate a general equilibrium model of a port city and examine the equilibrium conditions to identify the possible land use patterns. It will be shown that four land use patterns remain as possible ones after the elimination of the impossible ones. Above all, the new finding presented in this paper is the possiblity of a partially integrated land use pattern in which firms and households are partially integrated around the urban fringe. Since firms and households also form a monocentric land use pattern within the integrated zone, this pattern can be a new explanation of suburbanization of employment. Hence, to know exactly how this will happen is important from a theoretical point of view.

The content of this paper is organized as follows. In section 2, the assumptions of the model are formally stated. The behavior of firms and households is specified in terms of Cobb-Douglas type functions. This functional specification is adopted in view of mathematical tractability as well as flexibility to permit substitution among inputs (or consumer goods). With this specification, the bid rent functions of firms and households are derived taking into consideration the intraurban wage differentials, which are necessary for the compensation of extra commuting by households. The equilibrium conditions are formally stated by the use of these bid rent functions. In section 3 , an exhaustive examination of the equilibrium conditions reveals the possible land use patterns which will emerge in equilibrium. Then the task of proving the existence and uniqueness of each land use pattern will be lightened since we can now concentrate on a small number of cases. ${ }^{4}$. Finally, some concluding remarks follow in section 4.

\section{Model}

Since the concept of bid rent plays a central role in our model, the behavior of firms and households must be specific, and yet general enough for our analytical purpose. We adopt Cobb-Douglas type functional forms for production and utility functions. All the other assumptions adopted here seem common to the literature.

Assumption 1. The city is on a featureless plain with a single transport node (taken as the origin) through which all the products of firms are exported at a fixed price $p>0$.

Assumption 2. Households are homogeneous in taste and ability, and have the following utility function

$$
u(z, h)=z^{\alpha} h^{\beta}, \alpha, \beta>0, \alpha+\beta=1,
$$

4. See Koide (1989) for the details. 
where $z$ denotes the composite good consumption, and $h$ the amount of land consumed. The composite good is taken as the numeraire.

Assumption 3. All firms produce only one kind of product according to an identical production function

$$
f(K, L, S)=K^{a} L^{b} S^{c}, a, b, c>0, a+b+c=1,
$$

where $K$ denotes capital, $L$ labor, and $S$ land input.

Assumption 4. Transportation is possible in all directions, and the commuting cost for a household is given by $t d$, where $t>0$ represents the cost per unit distance and $d$ the distance traveled. The transport cost per unit of product for a firm located at distance $x$ from the transport node is given by $q x$, where $q>0$ is the cost per unit distance.

Assumption 5. The amount of land available for urban use at distance $x$ from the transport node is $2 \pi x$, and all land is owned by absentee landlords.

Assumption 6. The rental of capital is spatially fixed at $r>0$.

Assumption 7. The opportunity cost of land is zero.

Assumption 8. The city is open in the sense that migration between the city and the rest of the world is costless, and hence the utility level of the households in the city is exogenously given by $u$, which is determined in the rest of the world.

Since the specification of the production function implies constant returns to scale, we can embody the production technology of a firm at $x$ in the cost function per unit of output, $C[r, W(x), R(x)]=\min _{K, L, S}\{r K+W(x) L+R(x) S \mid f(K, L, S)=1\}$. The cost function depends on the rental of capital $r$, the wage rate $W(x)$ and the land rent $R(x)$, and can be expressed as

$$
C[r, W(x), R(x)]=a^{-a} b^{-b} c^{-c} r^{a} W(x)^{b} R(x)^{c} .
$$

Since competition leaves zero profit for all the firms irrespective of their locations, we have

$$
C[r, W(x), R(x)]=p-q x,
$$

which, by inversion, yields the bid rent function

$$
\Phi(x)=G(p-q x)^{\frac{1}{c}} W(x)^{-\frac{b}{c}},
$$

where

$$
G=a^{\frac{a}{c}} b^{\frac{b}{c}} c r^{-\frac{a}{c}} .
$$

The land requirement per unit of output associated with (3) is given by 


$$
s(x)=\frac{c(p-q x)}{\Phi(x)}=\frac{c}{G}(p-q x)^{-\frac{1-c}{c}} W(x)^{\frac{b}{c}} .
$$

That is, $s(x)$ is the land requirement for one unit of output when the land rent at $x$ is given by $\Phi(x)$.

On the other hand, a more careful derivation of the bid rent function of households is necessary since they can choose not only their residential location but also the location of their workplaces in consideration of the plausible wage differentials. Let $x_{W}$ denote the workplace of a household located at $x$. Then, the net income for the household is ${ }^{5}$

$$
I\left(x, x_{W}\right)=W\left(x_{W}\right)-t\left|x-x_{W}\right| .
$$

Now, using the indirect utility function $V(R, I)=\max _{z, h}\{u(z, h) \mid z+R h=I\}$, we can solve

$$
V\left[R(x), I\left(x, x_{W}\right)\right]=u
$$

for $R(x)$ and obtain the bid rent function of the household who lives at $x$ and commutes to $x_{W}$ as

$$
\phi\left(x, x_{W}\right)=K I\left(x, x_{W}\right)^{\frac{1}{\beta}}
$$

where

$$
K=\alpha^{\frac{\alpha}{\beta}} \beta u^{-\frac{1}{\beta}}
$$

Then, by defining

$$
I(x)=\max _{x_{w}} I\left(x, x_{w}\right),
$$

the bid rent in a strict sense, that is, the maximum land rent that a household can pay at $x$ while achieving the utility level $u$, can be expressed as

$$
\Psi(x)=K I(x)^{\frac{1}{\beta}} .
$$

The land consumption of the household associated with $\Psi(x)$ is given by

$$
h(x)=\frac{\beta I(x)}{\Psi(x)}=\frac{\beta}{K} I(x)^{-\frac{\alpha}{\beta}} .
$$

That is, $h(x)$ is the consumption of land which will result from the utility maximizing behavior of a household with net income $I(x)$ when the land rent at $x$ is given by $\Psi(x)$.

By assuming that the city has a property of circular symmetry, we can formally state the equilibrium conditions. Let $\theta(x)$ be the proportion of the total available land at $x$ that is occupied by households, and $\bar{x}$ be the urban fringe distance. Then, the equilibrium conditions that should determine $\{R(x), W(x), \theta(x), \bar{x}: 0 \leq x \leq \bar{x}\}$ can be stated as

5. When the commuting cost is paid by the employer, $t$ will be zero. But, in practice, household's utility also depends on the distance traveled, D, because of the disutility associated with commuting. Then the indirect utility function may be written as $\mathrm{V}(R, I, D)$, so the monetary evaluation of the marginal disutility of commuting is expressed as $\lambda=-(\partial \mathrm{V} / \partial D) /(\partial V / \partial I)$. If $\lambda$ is assumed to be constant, we have $V(R, I, D)=V(R, I-\lambda D, 0)$. Therefore, the discussion in the text can be maintained for the present case just by replacing $t$ in (5) with $\lambda$. 
follows :

i) land market conditions

$$
\begin{array}{ll}
R(x)=\max \{\Phi(x), \Psi(x), 0\}, & x \in[0, \bar{x}], \\
R(x)=\Phi(x) \text { if } \theta(x)<1, & x \in[0, \bar{x}], \\
R(x)=\Psi(x) \text { if } \theta(x)>0, & x \in[0, \bar{x}], \\
R(\bar{x})=0, &
\end{array}
$$

ii) labor market condition ${ }^{6}$

$$
\int_{0}^{\bar{x}} \frac{2 \pi x \theta(x)}{h(x)} d x=\int_{0}^{\bar{x}} \frac{b \Phi(x)}{c W(x)} 2 \pi x[1-\theta(x)] d x .
$$

(11) means that the equilibrium rent profile should be the upper envelope of the bid rent curves. (12) and (13) mean that firms and households should be able to pay the highest bid at their locations. (14) determines the urban fringe within which all the city activities occur. The left-hand side of (15) denotes the total supply of labor and the right-hand side, the total demand for labor. Note that $b \Phi(x) / c W(x)$ is the employment per unit of land. ${ }^{7}$

\section{Possible land use patterns}

It is convenient for our purpose to classify the urban area into three categories :
i) residential district $(R D)=\{x \mid \theta(x)=1,0 \leq x \leq \bar{x}\}$,
ii) production district $(P D)=\{x \mid \theta(x)=0,0 \leq x \leq \bar{x}\}$,
iii) integrated district $(I D)=\{x \mid 0<\theta(x)<1,0 \leq x \leq \bar{x}\}$.

Then we can define a land use pattern by the spatial distribution of the three kinds of districts defined above. There are three classes of land use patterns :

i) segregated land use pattern=land use pattern consisting solely of $R D$ 's and $P D$ 's,

ii) completely integrated land use pattern=land use pattern consisting solely of an $I D$,

iii) partially integrated land use pattern=land use pattern consisting of $R D$ 's, $P D$ 's and ID's.

To investigate the possible land use patterns in the model described in the previous section, we first focus on the nature of the wage function, $W(x)$. The following proposi-

6. To be exact, since (15) is only the aggregate condition for the labor market clearance, the condition must be stated so as to balance the demand and supply of labor at each location. This point will be properly taken into consideration in the following section.

7. To see this, recall the fundamental properties of a Cobb-Douglas production function ;

$$
S \Phi(X)=c C[r, W(X), R(X)]
$$

and

$$
L W(X)=b C[r, W(X), R(X)]
$$

where $S$ and $L$ are understood as the requirements of land and labor per unit of output. From these we can obtain the labor-land ratio $L / S$ as in (15).

8. For our analytical purpose, each district is well defined when it is connected and has a positive length. The boundary between two districts can be included in either district. 
tion is basic to the subsequent discussion.

Proposition 1. There is no outward commuting in equilibrium.

Proof. Suppose a household commutes from $x$ to $x^{\prime}\left(x<x^{\prime}\right)$ in equilibrium. Since the household maximizes its net income by commuting, it follows from (8) that

$$
W\left(x^{\prime}\right)-t\left(x^{\prime}-x\right) \geq W(x),
$$

that is,

$$
W\left(x^{\prime}\right)>W(x) .
$$

At $x^{\prime}, \Phi\left(x^{\prime}\right)$ must be at least as great as $\Psi\left(x^{\prime}\right)$, while $\Psi(x)$ is at least as great as $\Phi(x)$;

$$
\begin{gathered}
K W\left(x^{\prime}\right)^{\frac{1}{\beta}} \leq G\left(p-q x^{\prime}\right)^{\frac{1}{c}} W\left(x^{\prime}\right)^{-\frac{b}{c}}, \\
K W(x)^{\frac{1}{\beta}} \geq G(p-q x)^{\frac{1}{c}} W(x)^{-\frac{b}{c}} .
\end{gathered}
$$

From (17) and (18), we have

$$
K\left\{W(x)^{\frac{1}{\beta}}-W\left(x^{\prime}\right)^{\frac{1}{\beta}}\right\} \geq G\left\{(p-q x)^{\frac{1}{c}} W(x)^{-\frac{b}{c}}-\left(p-q x^{\prime}\right)^{\frac{1}{c}} W\left(x^{\prime}\right)^{-\frac{b}{c}}\right\} .
$$

From (16), the left-hand side of (19) is negative, while the right-hand side of (19) is positive (recall $x<x^{\prime}$ ). This is a contradiction.

Q.E.D.

The following proposition can be obtained immediately as a corollary.

Proposition 2. In equilibrium,

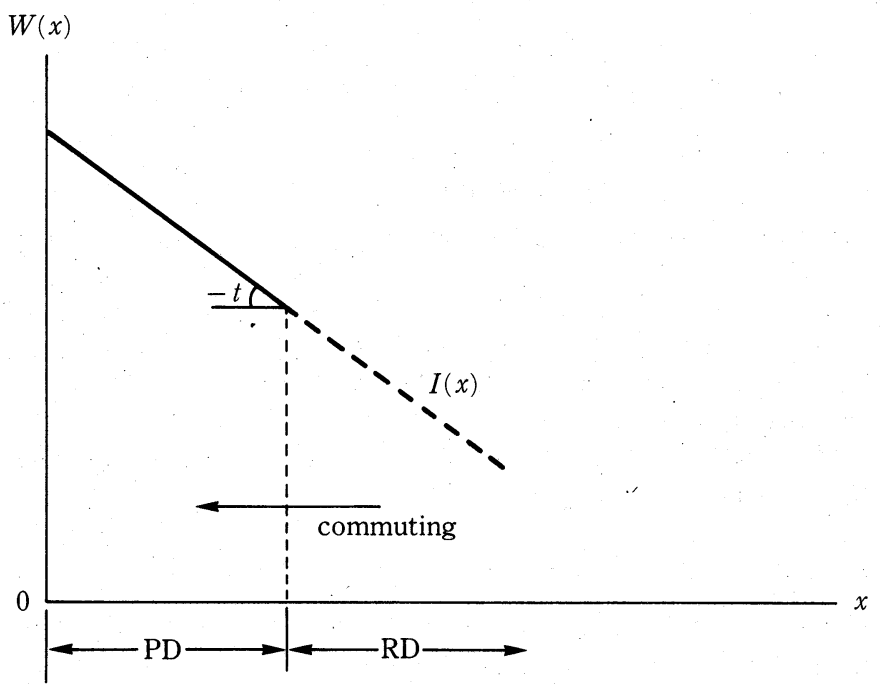

Figure 1 Wage Profile with Commuting. 
i) a production district cannot locate at the urban fringe, and

ii) a residential district cannot locate at the center.

Proposition 3. If commuting takes place in equilibrium, the wage rate $W(x)$ paid by firms should be a linear function with slope $-t$.

Proof. From Proposition 1, we have only to consider the case of inward commuting. Let $x$ and $x^{\prime}$ belong to an interval in which firms employ households who commute to them. For a household located at $a$ and commuting to $x(x<a)$, (8) implies

$$
W(x)-t(a-x) \geq W\left(x^{\prime}\right)-t\left(a-x^{\prime}\right),
$$

that is,

$$
W(x)-W\left(x^{\prime}\right) \geq-t\left(x-x^{\prime}\right) .
$$

Similarly, for another household located at $b$ and commuting to $x^{\prime}\left(x^{\prime}<b\right)$, the following holds ;

$$
W\left(x^{\prime}\right)-W(x) \geq-t\left(x^{\prime}-x\right) .
$$

From (20) and (21), we have

$$
W\left(x^{\prime}\right)-W(x)=-t\left(x^{\prime}-x\right),
$$

and by letting $x^{\prime} \rightarrow x$, we obtain ${ }^{9}$

$$
\frac{d W(x)}{d x}=-t
$$

Since $x$ is arbitrary, we have the desired result.

Q.E.D.

In Figure 1, a wage function is depicted for a monocentric case. Note that the extention of $W(x)$ onto the residential district (dotted line) gives the net income (i.e., disposable income for the composite good and land) realized at $x$. From the viewpoint of firms, they must pay land rent subject to the wage rate determined by the extended portion if they try to locate in the residential district. Thus, interpreting the wage function as indicating the net income realized at $x$, we can define $\Phi(x)$ and $\Psi(x)$ in any district. In particular, (9) and (10) are now rewrittten as

$$
\begin{aligned}
& \Psi(x)=K W(x)^{\frac{1}{\beta}} \\
& h(x)=\frac{\beta}{K} W(x)^{-\frac{\alpha}{\beta}} .
\end{aligned}
$$

In an integrated district, the wage function is generally not linear. Since $0<\theta(x)<1$ holds in an $I D$, the wage level at each location must be determined so that it is compatible with

9. If $x$ is an edge of the interval in question, $d W / d x$ should be understood as a one-sided derivative. 


$$
\Phi(x)=\Psi(x) \text { in an } I D .
$$

Using (3) and (22), we can solve (24) for $W(x)$ as

$$
W(x)=H(p-q x)^{\frac{\beta}{\beta b+c},}, \quad x \in\left[0, \frac{p}{q}\right),
$$

where

$$
H=\left(\frac{G}{K}\right)^{\frac{\beta c}{\beta b+c}}
$$

(25) implies

$$
W^{\prime}(x)=-\frac{\beta q}{\beta b+c} H(p-q x)^{\frac{\beta-\beta b-c}{\beta b+c}}<0, \quad x \in\left(0, \frac{p}{q}\right),
$$

and

$$
W^{\prime \prime}(x) \gtreqless 0 \quad \text { as } \quad \beta \gtrless \beta b+c \text {. }
$$

Proposition 4. Suppose the wage function is linear with slope $-t$ and $\Phi(x)=\Psi(x)$ for all $x \in[0, \bar{x})$ in equilibrium. Then the equilibrium is unstable in the sense that it can be transformed into another equilibrium with $u, \bar{x}, W(x)$ and $R(x)$ unchanged, in particular, into a completely integrated urban configuration without commuting.

Proof. From (25), the equilibrium wage function is

$$
W(x)=H(p-q x), x \in[0, \bar{x}) .
$$

Using (4) and (28), the employment per unit of land can be expressed as

$$
\frac{b \Phi(x)}{c W(x)}=\frac{b}{s(x) H} .
$$

Furthermore, using (10) and (28), we can express $h(x)$ as

$$
h(x)=\frac{\beta H(p-q x)}{\Psi(x)} .
$$

Substitution of (4) into the above relation with consideration of $\Phi(x)=\Psi(x)$ gives

$$
h(x)=\frac{\beta H}{c} s(x)
$$

Using (29) and (30), we can rewrite the labor market condition (15) as

$$
\int_{0}^{\bar{x}} \frac{2 \pi x \theta(x) c}{\beta s(x)} d x=\int_{0}^{\bar{x}} \frac{2 \pi x[1-\theta(x)] b}{s(x)} d x
$$

which, by the linearity of $W(x)$ (i.e., $\beta=\beta b+c$ ), is further simplified to

$$
\int_{0}^{\bar{x}} \frac{\theta(x) x}{s(x)} d x=\int_{0}^{\bar{x}} \frac{b x}{s(x)} d x .
$$

Since $W(x)$ is linear with slope $-t$, any household is indifferent to its residential location 
and those locations closer to the center when choosing its workplace. Therefore, (31) implies that even if $\theta(x)$ is changed to the constant, $b$, while maintaining the equilibrium values (functions), $\bar{x}, W(x)$ and $R(x)$, we will obtain another equilibrium. Or, as long as we maintain (31) and the equilibrium values (functions) except $\theta(x)$, and do not violate Proposition 1, we can change $\theta(x)$ as we like.

Next, we can show that $\theta(x)=b$ for all $x \in[0, \bar{x})$ is consistent with a completely integrated land use pattern without commuting. Suppose demand and supply of labor are balanced at each location. Then we have

$$
\frac{2 \pi x \theta(x)}{h(x)}=\frac{2 \pi x[1-\theta(x)] b}{s(x) H}
$$

Substitution of (30) into (32) gives

$$
(\beta b+c) \theta(x)=\beta b,
$$

which, in view of the linearity of $W(x)$, implies

$$
\theta(x)=b \text {. }
$$

Conversely, from (33) we can lead to (32).

Since a completely integrated land use pattern with $W^{\prime}(x)=-t, x \in(0, \bar{x})$ and its transformations have an amoebic pecularity due to our functional specification, they are not considered any further. We may call an equilibrium stable if it is not unstable.

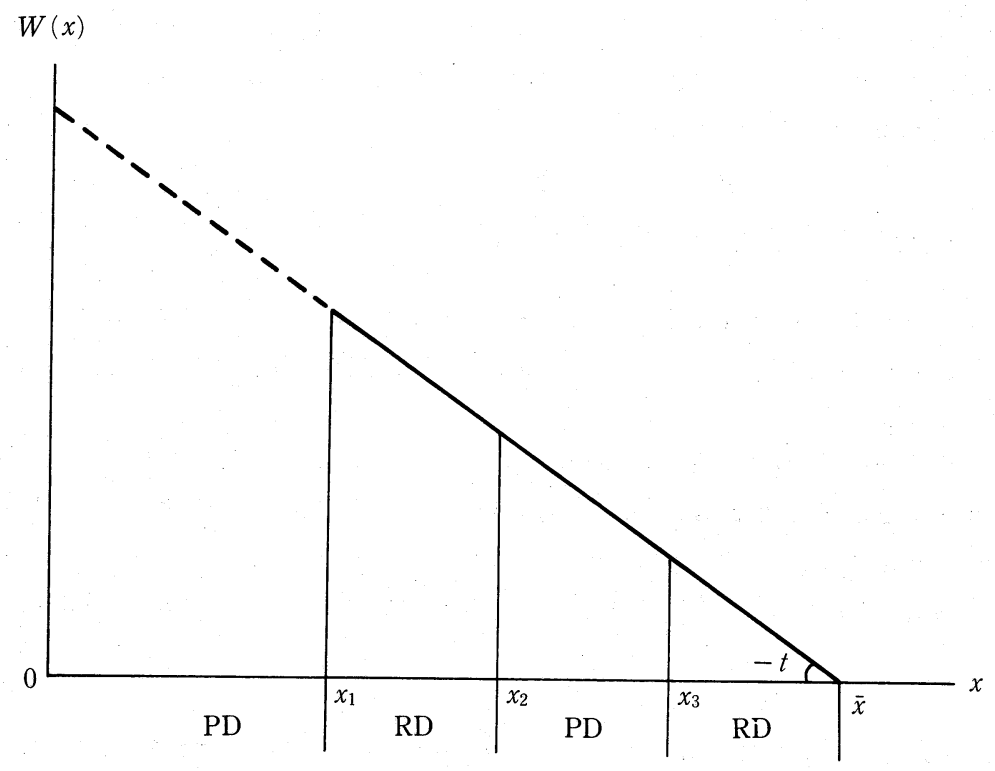

Figure 2 Wage Profile with more than One PD. 
Proposition 5. Segregated land use patterns with more than one production district cannot exist in stable equilibrium.

Proof. Suppose there exist more than one $P D$ and let the boundaries between $P D$ 's and $R D$ 's be as depicted in Figure 2, where we concentrate on the area close to $\bar{x}$. From (3) and (22), we have

$$
\begin{aligned}
& \frac{d \Phi(x)}{d x}=\Phi(x)\left\{-\frac{q}{c}(p-q x)^{-1}+\frac{b t}{c} W(x)^{-1}\right\}, \\
& \frac{d \Psi(x)}{d x}=\Psi(x)\left\{-\frac{t}{\beta} W(x)^{-1}\right\} .
\end{aligned}
$$

Observe the following :

$$
\begin{aligned}
\Phi\left(x_{i}\right) & =\Psi\left(x_{i}\right), \quad i=1,2,3, \\
\frac{d \Phi\left(x_{i}\right)}{d x} & \leq \frac{d \Psi\left(x_{i}\right)}{d x}, \quad i=1,3, \\
\frac{d \Phi\left(x_{2}\right)}{d x} & \geq \frac{d \Psi\left(x_{2}\right)}{d x} .
\end{aligned}
$$

These relations imply that the two expressions in the brackets of (34) and (35) must generally change their relative magnitudes at least twice between $x_{1}$ and $x_{3}$. Consequently, the following equation in $x$ must have at least two different solutions :

$$
-\frac{q}{c}(p-q x)^{-1}+\frac{b t}{c} W(x)^{-1}=-\frac{t}{\beta} W(x)^{-1} .
$$

But substitution of a linear function for $W(x)$ into the above equation gives only a linear equation in $x$, which has only one solution or infinitely many. In the former case, $\Phi(x)$ and $\Psi(x)$ cannot intersect more than twice, and there is no more than one $P D$ in equilibrium. In the latter case; $\Phi(x)$ and $\Psi(\dot{x})$ coincide entirely with $W^{\prime}(x)=-t$, which implies an unstable equilibrium by Proposition 4.

Q.E.D.

Proposition 6. In stable equilibrium, no households commute in any integrated district.

Proof. First, we consider the case $\beta \neq \beta b+c$. Suppose there is a household in an $I D$ who commutes. Then $W(x)$ has a linear portion in that $I D$, which, in view of (27), contradicts the assumption that $\beta \neq \beta b+c$.

Next, if $\beta=\beta b+c, W(x)$ is linear in any $I D$. Suppose there is a household in an $I D$ who commutes. Since the wage function must be continuous ( (otherwise, there will be an incentive for households to change their workplaces), $W^{\prime}(x)=-t$ for all $x \in(0, \bar{x})$, and hence $\Phi(x)=\Psi(x)$ for all $x \in[0, \bar{x})$. In view of Proposition 4 , this is a case of an unstable equilibrium.

Q.E.D.

Proposition 6 implies that each household in any integrated district must choose its workplace at its residential location in stable equilibrium. 


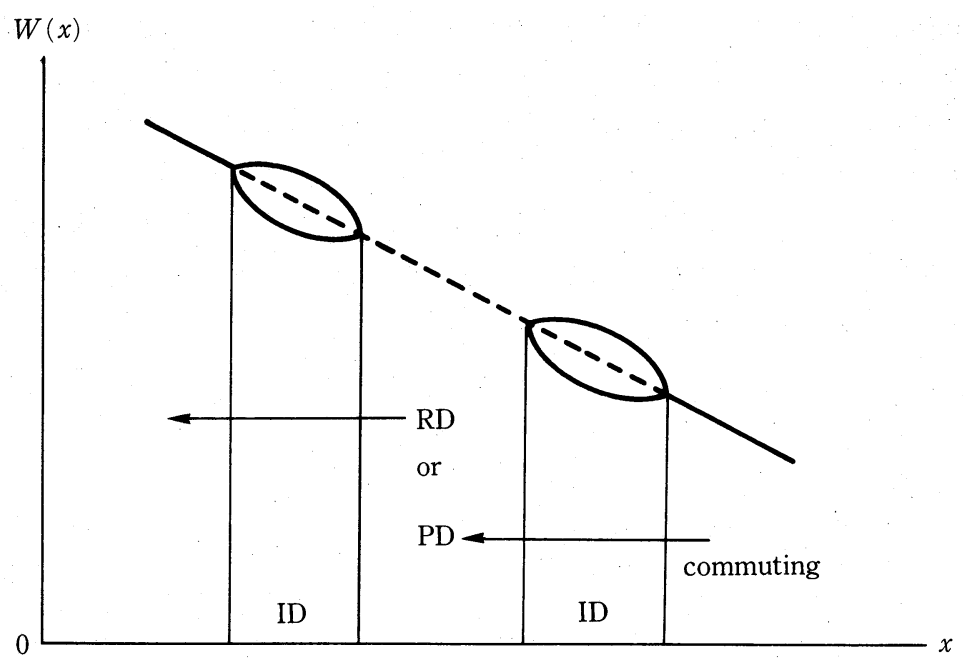

Figure 3 Wage Profile with Commuting beyond IDs.

Lemma 1. Neither a production district nor a residential district alone can locate between a pair of integrated districts in stable equilibrium.

Proof. Suppose the contrary, and first consider the case $\beta \neq \beta b+c$. Then, since $W(x)$ is strictly concave or strictly convex in $I D$ 's and $W(x)$ must be continuous, the wage profile observed by commuting households is a straight line and different from the actual one in ID's, as is depicted in Figure 3. This implies that some households can be better off by changing their workplaces. Hence, the original land use pattern cannot be in equilibrium.

Next, in the case $\beta=\beta b+c, W(x)$ becomes linear in $I D$ 's. Then it can be shown that $W(x)$ must be linear with slope $-t$ for all $x \in[0, \bar{x})$, which, in view of Proposition 4 , is the case of an unstable equilibrium.

Q.E.D.

Lemma 2. No partially integrated land use pattern with more than one integrated district can exist in stable equilibrium.

Proof. Suppose a partially integrated land use pattern with more than one $I D$ exists in equilibrium. Then by Lemma 1, there must be at least one $P D$ and one $R D$ between a pair of $I D$ 's for the equilibrium to be stable. But this is impossible since $\Phi(x)=\Psi(x)$ must hold for at least three different values of $x$ between the pair of $I D$ 's with a linear wage function (cf. the proof of Proposition 5).

Q.E.D.

Proposition 7. In stable equilibrium, a partially integrated land use pattern has one and only one integrated district, which occurs only when $\beta \neq \beta b+c$. If $\beta<\beta b+c$, the integrated district can locate only at the center. If $\beta>\beta b+c$, the integrated district can locate only at the urban fringe. 
Proof. By Lemma 2, there can be only one $I D$ in a stable equilibrium with a partially integrated land use pattern. Evidently, the $I D$ cannot locate strictly between 0 and $\bar{x}$. Otherwise, there is an incentive for households to change their workplaces (cf. Figure 3), or the equilibrium is unstable.

First, consider the case $\beta<\beta b+c$. In view of (27), this implies that $W(x)$ which equates $\Phi(x)$ and $\Psi(x)$ is strictly concave. Suppose the $I D$ locates at the urban fringe.

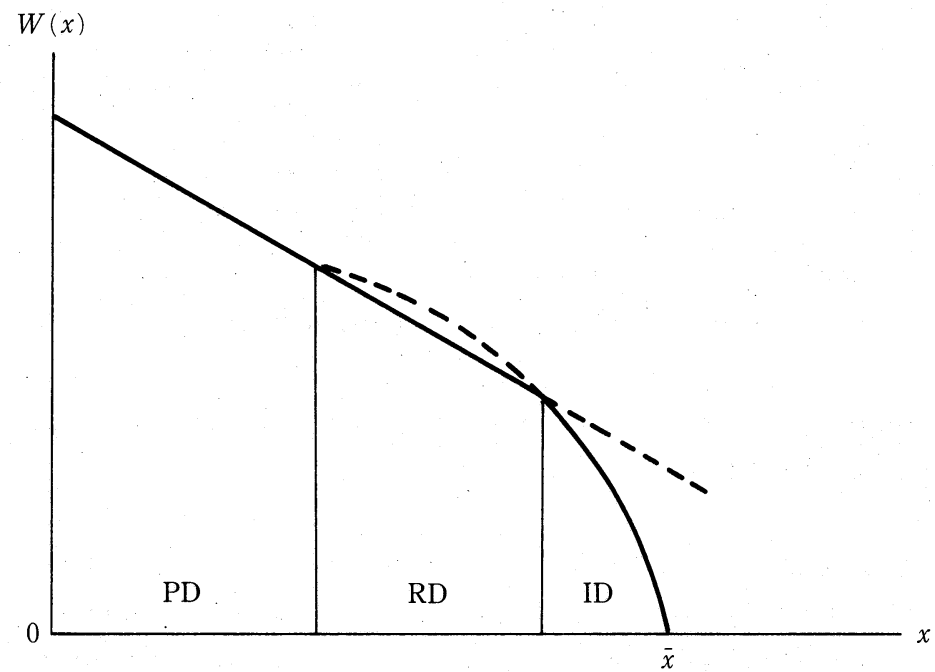

( a )

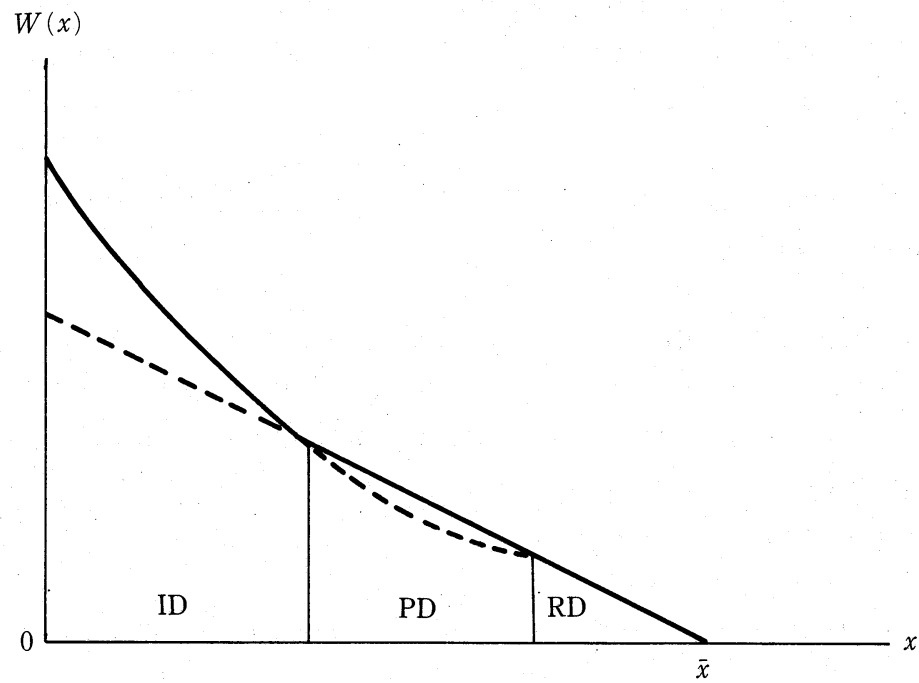

( b )

Figure 4 Wage Profile with an ID. 
By applying Proposition 5 to the inner side of the $I D$, it is shown that there must be one $P D$ and one $R D$ to the left of the $I D$, as depicted in Figure 4(a). Since $W(x)$ in the $I D$ is below the linear extension of $W(x)$ from the $R D$, the households in the $I D$ can be better off by working for the firms in the $P D$, which contradicts Proposition 6.

Next, consider the case $\beta>\beta b+c$. In this case, $W(x)$ which equates $\Phi(x)$ and $\Psi(x)$ is strictly convex. ${ }^{10}$ Suppose the $I D$ locates at the center, as depidcted in Figure 4(b). Since $W(x)$ in the $I D$ lies above the linear extension of $W(x)$ from the $P D$, the households in the $R D$ have an incentive to change their workplaces from the $P D$ to the $I D$. Thus, the original land use pattern cannot be in equilibrium.

Finally, consider the case $\beta=\beta b+c$. In this case, $W(x)$ in the $I D$ must be linear. Since $\Phi(x)=\Psi(x)$ must hold also at the boundary between the $P D$ and the $R D, W(x)$

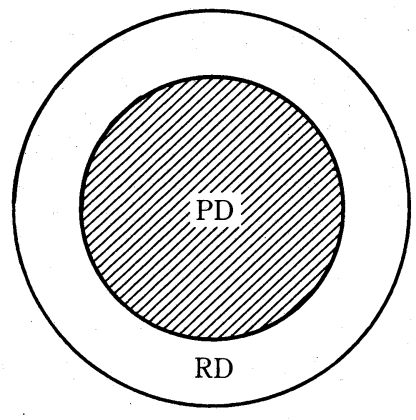

monocentric

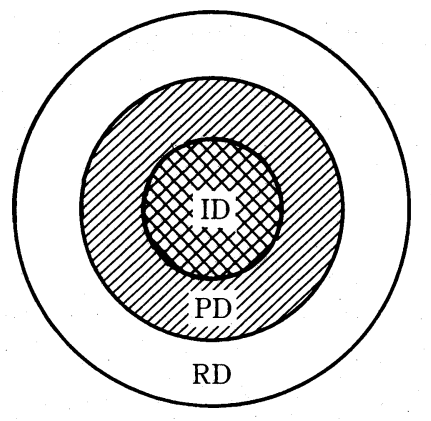

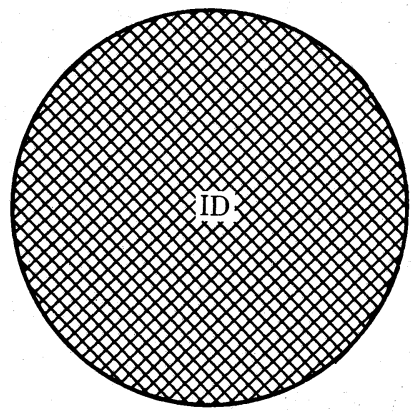

completely integrated

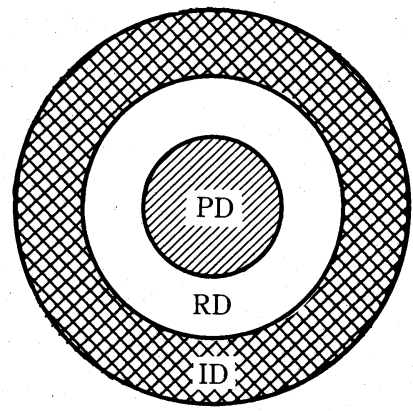

partially integrated

Figure 5 Possible Land Use Patterns.

10. Note that if the inputs are only labor and land, $\beta>\beta b+c$ never happens, for $\beta b+c$ is the weighted average of $\beta$ and 1 in this case. Thus, introduction of capital as an input is essential in our model to obtain a partially integrated land use pattern with an ID located at the urban fringe. 
must be linear with slope $-t$ between 0 and $\bar{x}$, which implies that the equilibrium is unstable.

Q.E.D.

To sum up, in stable equilibrium there are four types of possible land use patterns as depicted in Figure 5. In the category of segregated land use pattern, it follows from Proposition 5 that the only possible equilibrium land use pattern is the one consisting of one $P D$ surrounded by one $R D$, which can be appropriately called a monocentric land use pattern. By Proposition 7, there can be two types of partially integrated land use patterns, each of which has just one $I D$, according to the shape of $W(x)$ in the $I D$. A monocentric (completely integrated) land use pattern can be viewed as a special case of a partially integrated land use pattern in which the integrated district shrinks (spreads out) entirely.

\section{Concluding remarks}

In this paper, we shed light on the land use patterns which will be generated within the framework of a port city. Although the possible land use patterns are now limited to those in Figure 5, a final touch is necessary to complete our analysis, that is, we must prove the existence and uniqueness of each of the four land use pattern to assure that they have an actual meaning. This task will be carried out without much difficulty by making use of the wage function determined by (25). ${ }^{11}$

As for the variety of land use paterns, our model has no less explanatory power than Ogawa and Fujita (1980), but cannot generate such a land use pattern with subcenters as discussed by Fujita and Ogawa (1982). Therefore, to explain the urban spatial structure of modern business cities, it seems essential to theorize the complex interactions among agents as a source of urban concentration.

Finally, the seeming restrictiveness of Assumption 8 can be abated if we conssider the fundamental relation between open and closed models. Since the equilibrium utility in a closed model is endogenously determined for an exogenously given population, we can interpret the solution of an open model as the solution of a closed model by simply exchanging the roles of the utility level and the number of households.

\section{References}

[1] Alonso, William, Location and Land Use, Harvard University Press, 1964.

[ 2 ] Beckmann, Martin J., "Spatial Equilibrium in the Dispersed City," in G.J. Papageorgiou ed., Mathematical Land Use Theory, Lexington Books, 1976, pp. 117 125.

[ 3 ] Cappoza, Dennis R., "Employment-Population Ratio in Urban Areas: A Model of the Urban Land, Labor, and Goods Markets," in G.J. Papageorgiou ed., op. cit., pp. 127 143.

[4] Fujita, Masahisa, "Spatial Interactions and Agglomeration in Urban Economies," Working Paper in Regional Science and Transportation, No. 126, University of Pennsylvania, 1988.

[5] Fujita, Masahisa and Hideaki Ogawa, "Multiple Equilibria and Structural Transition of Non-

11. The proof proceeds in a simple constructive way by making use of the fact that $\Phi(x)>\Psi(x)$ if the actual wage rate at $x$ is lower than that given by (25) and vice versa. See Koide (1989) for the details. 
Monocentric Urban Configurations," Regional Science and Urban Economics, 12, 1982, pp. 161 $\sim 196$.

[6] Goldstein, Gerald S. and Leon N. Moses, "Interdependence and the Location of Economic Activities," Journal of Urban Economics, 2, 1975, pp. 63 84.

[7] Imai, Haruo, "CBD Hypothesis and Economies of Agglomeration," Journal of Economic Theory." 28, 1982, pp. 275 299.

[ 8 ] Koide, Hiroyuki, "General Equilibrium Analysis of Urban Spatial Structure : Port City Model Reconsidered," Working Paper in Regional Science and Transportation, No.129, University of Pennsylvania, 1989.

[9] Mills, Edwin S., Studies in the Structure of the Urban Economy, Johns Hopkins University Press, 1972.

[10] Muth, Richard F., Cities and Housing, University of Chicago Press, 1969.

[11] Ogawa, Hideaki and Masahisa Fujita, "Equilibrium Land Use Patterns in a Nonmonocentric City," Journal of Regional Science," 20, 1980, pp. 455 475.

[12] O'Hara, Donald J., "Location of Firms within a Square Central Business District," Journal of Political Economy, 85, 1977, pp. 1189 1207.

[13] Schweizer, Urs and Pravin Varaiya, "The Spatial Structure of Production with a Leontief Technology," Regional Science and Urban Economics, 6, 1976, pp. 231 251.

[14] Tauchen, Helen and Ann D. Witte, "An Equilibrium Model of Office Location and Contact Patterns," Environment and Planning A, 15, 1983, pp. 1311 1326.

[15]. Tauchen, Helen and Ann D. Witte, "Socially Optimal and Equilibrium Distributions of Office Activities: Models with Exogenous and Endogenous Contacts," Journal of Urban Economics, 15, 1984, pp. $66 \sim 86$.

[16] Wheaton, William C., "Monocentric Models of Urban Land Use : Contributions and Criticisms," in P. Mieszkowski and M. Straszheim eds., Current Issues in Urban Economics, Johns Hopkins University Press, 1979, pp. 107 129. 